\title{
Relationship Between Performance Improvement in Activities of Daily Living and Energy Intake in Older Patients With Hip Fracture Undergoing Rehabilitation
}

Hiroki Umezawa, BS ${ }^{1}$, Yoji Kokura, BS ${ }^{2}$, Satoko Abe, $\mathrm{PhD}^{3}$, Chieko Suzuki, MD, PhD ${ }^{4}$, Akiko Nishida, $\mathrm{BS}^{5}$, Yoshie Uchiyama, $\mathrm{BS}^{6}$, Keisuke Maeda, $\mathrm{MD}, \mathrm{PhD}^{7}$, Hidetaka Wakabayashi, $\mathrm{MD}, \mathrm{PhD}^{8}$, Ryo Momosaki, $\mathrm{MD} \mathrm{PhD}^{9}$

${ }^{1}$ Department of Physical Therapy, Geriatrics Research Institute and Hospital, Gunma; ${ }^{2}$ Department of Clinical Nutrition, Keiju Medical Center, Ishikawa; ${ }^{3}$ Department of Nursing, Showa University of Nursing and Rehabilitation Sciences,

Kanagawa; ${ }^{4}$ Department of Internal Medicine, Ajisu Kyoritsu Hospital, Yamaguchi; ${ }^{5}$ Department of Nutrition, Gotanda Rehabilitation Hospital, Tokyo; ${ }^{6}$ Department of Nursing, Shirakawa Kosei General Hospital, Fukushima; ${ }^{7}$ Department of Palliative and Supportive Medicine, Graduate School of Medicine, Aichi Medical University, Aichi; ${ }^{8}$ Department of Rehabilitation Medicine,

Yokohama City University Medical Center, Kanagawa; ${ }^{9}$ Department of Rehabilitation Medicine, Teikyo University School of Medicine University Hospital, Mizonokuchi, Kanagawa, Japan

Objective To analyze whether sufficient energy intake (EI) improves performance of activities of daily living (ADL) in patients with hip fracture admitted to rehabilitation hospitals. The adequate amount of EI for improving performance of ADL in patients with hip fracture remains unknown.

Methods This retrospective cohort study included all patients with hip fracture $(\mathrm{n}=234)$ admitted to rehabilitation hospitals in Japan. The inclusion criteria for this study were age $>65$ years and body mass index $<30.0 \mathrm{~kg} / \mathrm{m}^{2}$. Patients who were transferred to an acute hospital and those with missing case data were excluded. According to the amount of EI, the patients were classified into energy sufficiency and shortage groups (EI/total energy expenditure $\geq 1.0$ and $<1.0$, respectively). The Functional Independence Measure (FIM) and FIM gain were used to evaluate the patient disability level and change in patient status in response to rehabilitation. Finally, FIM gain was calculated as the discharge FIM score minus the admission FIM score.

Results The final analysis targeted 202 patients-53 (26.2\%) were in the energy shortage group and 149 (73.8\%) were in the energy sufficiency group. The energy sufficiency group had a greater FIM gain than the energy shortage group (mean, $25.1 \pm 14.2$ vs. $19.7 \pm 16.4 ; \mathrm{p}=0.024$ ). Furthermore, sufficient EI in the first week since admission $(\beta=0.165 ; 95 \%$ confidence interval, $0.392-5.230 ; p=0.023)$ was an independent factor of FIM gain.

Conclusion Among elderly patients with hip fracture admitted to rehabilitation hospitals in Japan, the amount of EI during the first week after admission was an independent factor of FIM gain.

Keywords Femoral fractures, Hospitals, Rehabilitation, Nutritional support, Recovery of function 


\section{INTRODUCTION}

Fundamentally speaking, malnutrition in patients with a hip fracture is a major problem that traditionally leads to poor patient outcomes. In a recent systematic literature review [1], the prevalence of malnutrition in patients with hip fracture was reported as $\mathbf{1 8 . 7 \%}$ when assessed using the Mini Nutritional Assessment (MNA) test. Also, when accounting for body mass index (BMI) and weight loss, the percentage of malnutrition was noted at $45.7 \%$ in those patients reviewed. For this reason, malnutrition leads to many problems for the patient, including postoperative complications [2,3], poor functional improvement [4,5], longer hospital stays [2], high readmission rate [2], and the incidence of a high mortality rate $[2,6]$. Recently, it has been suggested that improving the nutritional status of this patient population can independently and significantly improve their physical function [7]. This is an important revelation, considering that $18.7 \%-45.7 \%$ of patients with fractured hip suffer from malnutrition, improving the nutritional status of these individuals will greatly improve their overall health and well-being.

Previous reports from rehabilitation hospitals in Japan suggest that in patients with hip fracture, the incidence of an improvement of the status of malnutrition in the patient is an independent factor that leads to an improved ability to perform activities of daily living (ADL). However, it remains unknown whether this effect also applies to elderly patients who have an experience with hip fracture at rehabilitation hospitals. The study of Goisser et al. [4] reported that dietary intake during the 4 days following the operation affected the improvement of ADL at 6 months after the surgery. Furthermore, Inoue et al. [5] reported that when evaluating the improvement of ADL according to the efficiency of motor Functional Independence Measure (FIM) gain, energy intakes (EIs) during the first week postoperatively were an independent factor that impacted the patient's health. It is noted that the rehabilitation hospital in Japan performs intensive rehabilitation after acute treatment and rehabilitation is provided for 2-3 hours daily [8]. It is noted that at rehabilitation hospitals, the activity of patients with hip fracture is higher when compared to the acute treatment ward. Therefore, the measured energy consumption of patients with hip fracture is higher, and has a greater caloric requirement than those in the acute phase. Nii et al. [9] reported that higher EI significantly improved the ADL in patients with stroke in rehabilitation hospitals. However, the evaluated adequate amount of EI for improving their ADL in patients with hip fracture remains unknown.

We aimed to retrospectively analyze whether sufficient EI improves ADL in patients with hip fracture in rehabilitation hospitals.

\section{MATERIALS AND METHODS}

\section{Study design}

This retrospective cohort study utilized the Japanese Rehabilitation Nutrition Database (JRND). In effect, the JRND is a large-scale database open for clinical research on rehabilitated nutrition [10]. The participants of the present study were all hip fracture patients admitted to the rehabilitation hospital in Japan who are registered in JRND. As a note, a rehabilitation hospital is a hospital where patients undertake rehabilitation for approximately 1-3 hours every day for a period ranging from 1 to 4 months, with the purpose of improving their functional capacity and returning home. The inclusion criteria in this study were age over 65 years old and a BMI less than $30.0 \mathrm{~kg} / \mathrm{m}^{2}$. We excluded the subjects who were transferred to an acute hospital and those with any missing case data. In this case, all subjects were followed up on until they were discharged. Informed consent was waived because of the anonymous nature of the data. The present study was approved by the Ethics Committee at the Jikei University School of Medicine (No. 27-150-[8035]).

The patient basic information, such as the age, sex, BMI, type of fracture, and surgical procedure was obtained from the database. The type of fracture was also therefore classified into femoral neck and trochanteric. The surgical procedures were classified into osteosynthesis, femoral head replacement, and other orthopedic surgery. Chiefly, the Charlson Comorbidity Index (CCI) [11] and certification for public long-term care insurance (LTCI) before hip fracture were used for analysis. Furthermore, intervals between the onset and admission, FIM score at both admission and discharge point, and the average amount of rehabilitation per day were obtained. Additionally, the Mini Nutritional Assessment (MNA) Short-Form [12], total energy expenditure (TEE), and EI on admission were acquired.

The LTCI is a public social security service in Japan, and 
all users are certificated into seven levels of care after a dedicated assessment.

\section{El and energy sufficiency}

In this study, the EI was calculated by averaging the energy provided from oral, intravenous, and enteral nutrition for 7 days after admission. Additionally, the oral EI was evaluated by a nurse or registered dietitian at each ward. Whereas an evaluation of the amount of dietary intake and the method used to calculate EI were not standardized, the visual assessment method is common in Japan, and nurses or registered nutritionists routinely evaluate the proportion of meals after each meal. These professionals also routinely calculate EIs from the leftovers and the amount of energy offered. Hence, the EI was calculated three times a day. Notably, the EI by intravenous and enteral nutrition used as per the doctor's directions was also recorded.

In this case, each patient's basal energy expenditure (BEE) was calculated using the Harris-Benedict formula [13]. In the calculation, we used the ideal body weight for patients. Going further, we estimated the TEE by multiplying BEE with a stress factor of 1.1 and an activity factor of 1.2. Incidentally, the setting of these TEEs was selected according to suggestions made by Inoue et al. [5]. The subjects were classified into an energy sufficiency group and an energy shortage group based on the amount of EI. Here, we defined the 'energy sufficiency group' with an EI/TEE of $\geq 1.0$ and 'energy shortage group' $<1.0$.

\section{Outcome measurement}

In this analysis, we used FIM to evaluate patient disability level as well as a change in patient status in response to rehabilitation. FIM is composed of 13 motor scales and 5 cognitive scales [14]. This system works and sorts the functional status of a person based on the level of assistance from 1 (total assistance) to 7 (complete independence). Accordingly, the total FIM scores ranged from 18 to 126 . FIM gain was calculated as the patient's discharge FIM score minus the admission FIM score. In this case, the FIM gain shows improvement in ADL during hospitalization. To some degree with a larger score, we can determine that the progression of ADL has largely improved.

\section{Statistical analysis}

In this study, all statistical analyzes were performed using IBM SPSS version 23.0 for Windows (IBM Corporation, Armonk, NY, USA). Notably, the continuous data are presented as mean \pm standard deviation and nonparametric data as the median (interquartile range [IQR] 25-75 percentile). In this context, the differences were analyzed using the Student t-test and Mann-Whitney U-test after confirming the normal distribution. The categorical data were expressed as incidences and percentages, with comparisons carried out using the chi-square test. The correlation analyses were carried out using Spearman rank correlation coefficients for age, CCI, number of days between onset and admission, a period of rehabilitation (min/day), FIM score at admission, and FIM gain. Also, the explanatory variable used in the multiple regression analysis was selected with reference to that reported previously $[5,7]$. In this case, the selected factors were based on the following eight items: age, sex, CCI, number of days from onset to the admission, the presence of surgical procedure, period of rehabilitation, FIM score at admission, and presence of certification for LTCI before hip fracture. Namely, the multicollinearity was assessed using the variance inflation factor (VIF) coefficient. Additionally, the multicollinearity was judged when the VIF was $\geq 2$. Throughout, $p$-values of $<0.05$ were considered statistically significant.

\section{RESULTS}

In this case, there were 234 patients with hip fracture were registered with the database from November 2015 to March 2018. Notably, there were 226 patients who were included in the final analysis. Thus, out of 24 patients, 7 transferred to the acute hospital, while 17 with insufficient data were excluded. The final analysis targeted 202 patients.

To begin with, Table 1 shows the baseline characteristics of the study participants. Accordingly, of the 202 patients, 43 were males and 159 females. The mean age was $84.9 \pm 7.4$ years. In this study, there were 108 patients with femoral neck fracture (53.5\%) and 94 patients with trochanteric fracture $(46.5 \%)$. The surgical procedure with 111 patients $(55.0 \%)$ included osteosynthesis, and 74 patients $(36.6 \%)$ underwent femoral head replacement. Ultimately, the median number of days (IQR 25-75 
Table 1. Patient characteristics

\begin{tabular}{|c|c|c|c|c|}
\hline Characteristic & All $(n=202)$ & $\begin{array}{c}\text { Energy shortage } \\
\text { group }(n=53)\end{array}$ & $\begin{array}{l}\text { Energy sufficiency } \\
\text { group }(n=149)\end{array}$ & p-value \\
\hline Age (yr) & $84.9 \pm 7.4$ & $82.1 \pm 7.6$ & $85.9 \pm 7.0$ & $0.001^{a)}$ \\
\hline Sex, female & $159(78.7)$ & $38(71.7)$ & $121(81.2)$ & $0.146^{\mathrm{b})}$ \\
\hline \multicolumn{5}{|l|}{ Type of fracture } \\
\hline Femoral neck & $108(53.5)$ & $31(58.5)$ & $77(51.7)$ & $0.393^{\mathrm{b})}$ \\
\hline Trochanteric & $94(46.5)$ & $22(41.5)$ & $72(48.3)$ & \\
\hline \multicolumn{5}{|l|}{ Surgical procedure } \\
\hline Osteosynthesis & $111(55.0)$ & $25(47.2)$ & $88(57.7)$ & $0.604^{\mathrm{b})}$ \\
\hline Femoral head replacement & $74(36.6)$ & $23(43.4)$ & $53(34.2)$ & \\
\hline Others & $6(3.0)$ & $2(3.8)$ & $4(2.7)$ & \\
\hline Nonsurgical treatment & $11(5.4)$ & $3(5.7)$ & $8(5.4)$ & \\
\hline Charlson comorbidity index & $1(0-2)$ & $1(0-2)$ & $1(0-2)$ & $0.550^{\mathrm{c})}$ \\
\hline Certification for LTCI before hip fracture & $93(45.1)$ & $30(56.6)$ & $63(42.3)$ & $0.072^{\mathrm{b})}$ \\
\hline Days between onset and admission & $22(18.0-30.0)$ & $21(16.0-33.5)$ & $22(18.0-30.0)$ & $0.493^{\mathrm{c})}$ \\
\hline \multicolumn{5}{|l|}{ FIM score } \\
\hline Admission & $70.3 \pm 25.4$ & $66.6 \pm 29.4$ & $71.7 \pm 23.7$ & $0.209^{a)}$ \\
\hline Discharge & $94.1 \pm 26.9$ & $86.3 \pm 31.0$ & $96.8 \pm 24.7$ & $0.014^{\mathrm{a})}$ \\
\hline FIM gain & $23.8 \pm 15.0$ & $19.7 \pm 16.4$ & $25.1 \pm 14.2$ & $0.024^{\mathrm{a})}$ \\
\hline Period of rehabilitation (min/day) & $104.3 \pm 29.5$ & $104.4 \pm 25.6$ & $104.3 \pm 30.8$ & $0.996^{\mathrm{a})}$ \\
\hline $\mathrm{BMI}\left(\mathrm{kg} / \mathrm{m}^{2}\right)$ & $20.1 \pm 3.1$ & $19.5 \pm 3.0$ & $20.3 \pm 3.1$ & $0.096^{\mathrm{a})}$ \\
\hline MNA Short-Form & $6.0 \pm 2.2$ & $5.6 \pm 2.2$ & $6.1 \pm 2.2$ & $0.124^{\mathrm{a})}$ \\
\hline TEE (kcal/IBW/day) & $1,212 \pm 208$ & $1,301 \pm 222$ & $1,180 \pm 195$ & $<0.001^{\text {a) }}$ \\
\hline Energy intake & $1,304 \pm 274$ & $1,024 \pm 290$ & $1,404 \pm 186$ & $<0.001^{a)}$ \\
\hline Energy intake (kcal/kg/day) & $29.9 \pm 7.7$ & $22.8 \pm 6.5$ & $32.4 \pm 6.3$ & $<0.001^{\text {a) }}$ \\
\hline
\end{tabular}

Values are presented as mean \pm standard deviation or number (\%) or median (interquartile range).

LTCI, public long-term care insurance; FIM, Functional Independence Measure; BMI, body mass index; MNA, Mini Nutritional Assessment; TEE, total energy expenditure.

${ }^{\text {a) }}$ Student t-test.

${ }^{\text {b) }}$ Chi-square test.

${ }^{c}$ Mann-Whitney U-test.

percentile) from the onset of an injury to entering a rehabilitation hospital was 22 (18.0-30.0). Chiefly, 53 patients $(26.2 \%)$ were in the energy shortage group, and 149 (73.8\%) were in the energy sufficiency group. In comparison to the energy shortage group, the sufficiency group was significantly older $(\mathrm{p}=0.001)$ but also had a higher FIM score at discharge $(\mathrm{p}=0.014)$, and EI $(\mathrm{p}<0.001)$. For this reason, the TEE was significantly higher in the shortage group $(\mathrm{p}<0.001)$.

Next, Table 2 shows the results of the univariate analysis for FIM gain. Hence, as compared to the energy sufficiency group, the shortage group $(\mathrm{p}=0.024)$ were more impaired in FIM gain.
In short, Table 3 shows correlation analysis results of age, CCI, number of days from onset to admission, period of rehabilitation (min/day), FIM score at admission, and FIM gain. There was a significant correlation between FIM gain and FIM score at admission $(\rho=-0.185$, $\mathrm{p}<0.001)$.

Finally, Table 4 shows the results of multiple regression analysis for the FIM gain. Collinearity was not observed in all items in the multiploidization test using VIF. As a result, the energy sufficiency group in the first week since admission $(\beta=0.165 ; 95 \%$ confidence interval [CI], 0.392 to $5.230 ; \mathrm{p}=0.023$ ) and the FIM score at admission ( $\beta=-0.304 ; 95 \% \mathrm{CI},-0.279$ to $-0.080 ; \mathrm{p}=0.001)$ were inde- 
pendent factors for FIM gain.

\section{DISCUSSION}

It is important to realize that the primary original finding of this investigation was two-fold. First, in elderly patients with hip fracture in rehabilitation hospitals, the amount of EI during the first week after admission was revealed to be an independent factor for FIM gain. Secondly, $26.2 \%$ of these patients were found to be caloric deficient.

Table 2. Univariate analysis of FIM gain

\begin{tabular}{|c|c|c|}
\hline Characteristic & FIM gain & p-value \\
\hline \multicolumn{3}{|l|}{ Sex } \\
\hline Male & $23.1 \pm 13.4$ & $0.747^{\mathrm{a})}$ \\
\hline Female & $23.9 \pm 13.9$ & \\
\hline \multicolumn{3}{|l|}{ Type of fracture } \\
\hline Femoral neck & $23.2 \pm 13.9$ & $0.597^{\mathrm{a})}$ \\
\hline Trochanteric & $24.3 \pm 16.1$ & \\
\hline \multicolumn{3}{|l|}{ Surgery } \\
\hline Presence & $23(12-32)$ & $0.601^{\mathrm{b})}$ \\
\hline Absence & $23(14-36)$ & \\
\hline \multicolumn{3}{|c|}{ Certification for LTCI before hip fracture } \\
\hline Presence & $23.3 \pm 14.7$ & $0.688^{\mathrm{a})}$ \\
\hline Absence & $24.1 \pm 15.2$ & \\
\hline \multicolumn{3}{|l|}{ Energy sufficiency } \\
\hline Presence & $25.1 \pm 14.2$ & $0.024^{\mathrm{a})}$ \\
\hline Absence & $19.7 \pm 16.4$ & \\
\hline
\end{tabular}

Values are presented as mean \pm standard deviation or median (interquartile range).

FIM, Functional Independence Measure; LTCI, public long-term care insurance.

${ }^{\text {a) }}$ Student t-test.

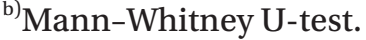

The most notable finding of this investigation was that the amount of EI during the first week after admission was an independent factor for FIM gain. The study of Inoue et al. [5] reported that EIs during the first week after the operation was an independent factor for improving motor FIM gain efficiency. Furthermore, Goisser et al. [4] reported that the number of dietary intakes during 4 days after operation affected the improvement of ADL at 6 months after surgery. Although the study design used herein is quite different from previous reports, the results are similar in spite of where the amount of EI affected the improvement of ADL. Then again, there were several investigations that have also reported that concomitant use of dietary supplements in addition to a healthy diet improves muscle mass, grip strength, and ADL [15-17]. In a review of the previous studies, it has been recognized that the muscular strength (grip strength) is strong when the intake energy is high $[18,19]$. Furthermore, an RCT at the rehabilitation hospitals showed that increased EI with oral nutritional supplements ingestion improved the patient grip strength $[17,20]$. Therefore, we did not measure muscle strength to investigate the relation between EI satisfaction and grip strength in our study. Also, it is significant to note that the shortage of EI caused a poor improvement in nutritional status, which resulted in an insufficient ADL improvement. Based on the results of the present study, to improve ADL in patients with hip fracture, it is essential for the medical staff to evaluate whether the EI of the patient is adequate. Nonetheless, while the optimal amount of EI remains unknown, the amount of daily activity and patient's condition will affect their EI, even if we assume IE/TEE $>1.0$ as a standard figure.

Equally important, it is also worth mentioning that $26.2 \%$ of the elderly patients with hip fracture in reha-

Table 3. Spearman rank correlation coefficients among the factors

\begin{tabular}{lccccc}
\hline & $\begin{array}{c}\text { Charlson } \\
\text { comorbidity } \\
\text { index }\end{array}$ & $\begin{array}{c}\text { Days from } \\
\text { onset to } \\
\text { admission }\end{array}$ & $\begin{array}{c}\text { Period of } \\
\text { rehabilitation } \\
\text { (min/day) }\end{array}$ & $\begin{array}{c}\text { FIM score at } \\
\text { admission }\end{array}$ & FIM gain \\
\hline Age & 0.070 & -0.083 & -0.084 & $-0.351^{*}$ & 0.113 \\
Charlson comorbidity index & & 0.095 & 0.051 & $-0.225^{*}$ & -0.037 \\
Days from onset to admission & & & -0.028 & -0.022 & -0.016 \\
Period of rehabilitation (min/day) & & & & 0.060 & 0.048 \\
FIM score at admission & & & & & $-0.185^{*}$ \\
\hline
\end{tabular}

FIM, Functional Independence Measure.

${ }^{*} \mathrm{p}<0.005$. 
Table 4. Multivariate analysis of FIM gain

\begin{tabular}{lcrrrr} 
& \multirow{2}{*}{ p-value } & $\beta$ & \multicolumn{2}{c}{$\mathbf{9 5 \%}$ CI } & \multirow{2}{*}{ VIF } \\
\cline { 5 - 6 } & & & Lower & Upper & \\
\hline Age & 0.960 & -0.004 & -0.325 & 0.309 & 1.329 \\
Sex & 0.934 & 0.006 & -2.532 & 2.756 & 1.145 \\
Charlson comorbidity index & 0.812 & -0.018 & -2.217 & 1.739 & 1.192 \\
Days from onset to admission & 0.245 & -0.082 & -0.270 & 0.069 & 1.041 \\
Surgical procedure, presence & 0.546 & -0.043 & -6.025 & 3.199 & 1.070 \\
Period of rehabilitation & 0.361 & 0.064 & -0.038 & 0.103 & 1.039 \\
FIM score at admission & 0.001 & -0.304 & -0.279 & -0.080 & 1.556 \\
Certification for LTCI before hip fracture, presence & 0.066 & -0.152 & -4.717 & 0.156 & 1.441 \\
Energy sufficiency, presence & 0.023 & 0.165 & 0.392 & 5.230 & 1.106 \\
\hline
\end{tabular}

FIM, Functional Independence Measure; LTCI, long-term care insurance; CI, confidence interval.

bilitation hospitals were in the energy shortage state, as compared to $82.5 \%$ in the acute phase hospital during the first week after the operation, with an average EI amount of $933.0 \mathrm{kcal}$ (quartile 806.9-1,120.1) [5]. Also, for one thing $71.5 \%$ of the patients took less than $50 \%$ of their offered meal during the 4 days after the operation [4]. Furthermore, in patients in rehabilitation for cerebrovascular disorders, the average EI amount during 3 days after admission was $33.2 \mathrm{kcal} / \mathrm{kg} /$ day (quartile 29.2-39.75) [9].

Even though the fraction or percentage of the EI shortage in our study was $26.2 \%$, it is less than that reported in acute phase hospitals. However, the EI amount of $29.9 \pm 7.7 \mathrm{kcal} / \mathrm{kg} /$ day was less than that of patients with cerebrovascular disease disorders. Whereas in the present investigation, the cause of short EI remains unknown. While in previous studies, factors of the EI shortage of hip fracture patients include changes in sensory organs, loss of a tooth, lack of primary caregivers, and in some cases, adverse effects of certain drugs [1]. These factors may have also contributed to the lower dietary intake observed in the present investigation. Despite the notation that as 1 in 4 elderly patients with hip fracture in rehabilitation hospitals may have fallen into energy shortage, it is vital to evaluate EI during the first week since admission and provide nutritional support to promote the best patient outcomes in this case.

In particular, sufficient EI and appropriate rehabilitation are essential for ADL improvement in patients with hip fracture in rehabilitation hospitals. In these cases, the combination of rehabilitation and nutrition care is called rehabilitation nutrition. In this concept, both rehabilitation and nutrition management is performed together with the International Classification Guidelines on Dysfunction and Health (ICF) to evaluate the subject's nutritional status and maximize the function of the elderly and disabled [21-23]. Moreover, as nutritional evaluation, intervention, and rehabilitation are performed concurrently; it has been surmised that rehabilitation nutrition is useful for ADL improvement in patients with hip fracture in rehabilitation hospitals.

With this in mind, we considered that the mean gain in FIM (FIM score at discharge - FIM score on admission) is greatest in patients with moderate assistance, whereas patients with low FIM scores on admission show little improvement. Because of its ceiling effects, those with high FIM scores on admission, who require minimal assistance, will subsequently have little gain in FIM [24].

Notwithstanding, there are several limitations to the present research. First, the energy ingestion methods (oral, intravenous, tube proportion) used in the included subjects were noted as unknown. Second, EI was evaluated only during the first week after the admission of the patient. In addition, the hospitalization period at the rehabilitation hospitals tends to become longer than that observed in the experience of using acute phase care facilities. Third, we did not measure the patient muscular strength in the present study. Therefore, it may be necessary to consider the length and stage of the investigation when determining results of the data.

Nevertheless, in this investigation, the EI of elderly patients with hip fracture in rehabilitation hospitals was an independent factor for FIM gain, and $26.2 \%$ of them were considered to be in an EI shortage state. Even so the present study showed that ADL could be improved more 
effectively by improving the nutritional status of the patients (IE/TEE >1.0).

\section{CONFLICT OF INTEREST}

No potential conflict of interest relevant to this article was reported.

\section{ACKNOWLEDGMENTS}

We thank the staff of the following hospitals for providing the patient's data for the Japanese Rehabilitation Nutrition Database (JRND): Atagawa Hospital, Nagasaki Rehabilitation Hospital, Miharu Hospital, Tsurumaki Onsen Hospital, Haradoi Hospital, Nanko Hospital, NishiHiroshima Rehabilitation Hospital, Yasuoka Hospital, Kanazawa Nishi Hospital, Sakurakai Medical Corporation Sakurakai Hospital, Sapporo Nishi-maruyama Hospital, Mihono Hospital, Tamana Regional Health Medical Center, Jikei University Daisan Hospital, Nakanoshima Iwaki Hospital, Nishinomiya Kyoritsu Neurosurgical Hospital, and Minamisoma Municipal General Hospital.

\section{AUTHOR CONTRIBUTION}

Conceptualization: all authors. Methodology: all authors. Formal analysis: Umezawa H, Kokura Y, Abe S, Suzuki C, Nishida A, Uchiyama Y. Funding acquisition: Momosaki R. Project administration: Maeda K, Wakabayashi H, Momosaki R. Visualization: Kokura Y, Abe S. Writing original draft: Umezawa H, Kokura Y, Abe S, Suzuki C. Writing - review and editing: Umezawa $\mathrm{H}$, Maeda $\mathrm{K}$, Wakabayashi H, Momosaki R. Approval of final manuscript: all authors.

\section{REFERENCES}

1. Malafarina V, Reginster JY, Cabrerizo S, Bruyere O, Kanis JA, Martinez JA, et al. Nutritional status and nutritional treatment are related to outcomes and mortality in older adults with hip fracture. Nutrients 2018; 10:E555.

2. Bohl DD, Shen MR, Hannon CP, Fillingham YA, Darrith B, Della Valle CJ. Serum albumin predicts survival and postoperative course following surgery for geriatric hip fracture. J Bone Joint Surg Am 2017;99:2110-8.
3. Mazzola P, Ward L, Zazzetta S, Broggini V, Anzuini A, Valcarcel B, et al. Association between preoperative malnutrition and postoperative delirium after hip fracture surgery in older adults. J Am Geriatr Soc 2017; 65:1222-8.

4. Goisser S, Schrader E, Singler K, Bertsch T, Gefeller O, Biber R, et al. Low postoperative dietary intake is associated with worse functional course in geriatric patients up to 6 months after hip fracture. Br J Nutr 2015; 113:1940-50.

5. Inoue T, Misu S, Tanaka T, Sakamoto H, Iwata K, Chuman $\mathrm{Y}$, et al. Inadequate postoperative energy intake relative to total energy requirements diminishes acute phase functional recovery from hip fracture. Arch Phys Med Rehabil 2019;100:32-8.

6. Miyanishi K, Jingushi S, Torisu T. Mortality after hip fracture in Japan: the role of nutritional status. J Orthop Surg (Hong Kong) 2010;18:265-70.

7. Nishioka S, Wakabayashi H, Momosaki R. Nutritional status changes and activities of daily living after hip fracture in convalescent rehabilitation units: a retrospective observational cohort study from the japan rehabilitation nutrition database. J Acad Nutr Diet 2018; 118:1270-6.

8. Miyai I, Sonoda S, Nagai S, Takayama Y, Inoue Y, Kakehi A, et al. Results of new policies for inpatient rehabilitation coverage in Japan. Neurorehabil Neural Repair 2011;25:540-7.

9. Nii M, Maeda K, Wakabayashi H, Nishioka S, Tanaka A. Nutritional improvement and energy intake are associated with functional recovery in patients after cerebrovascular disorders. J Stroke Cerebrovasc Dis 2016;25:57-62.

10. Takasaki M, Momosaki R, Wakabayashi H, Nishioka S. Construction and quality evaluation of the Japanese rehabilitation nutrition database. J Nutr Sci Vitaminol (Tokyo) 2018;64:251-7.

11. Charlson ME, Pompei P, Ales KL, MacKenzie CR. A new method of classifying prognostic comorbidity in longitudinal studies: development and validation. J Chronic Dis 1987;40:373-83.

12. Guigoz Y, Lauque S, Vellas BJ. Identifying the elderly at risk for malnutrition. The Mini Nutritional Assessment. Clin Geriatr Med 2002;18:737-57.

13. Long CL, Schaffel N, Geiger JW, Schiller WR, Blakemore WS. Metabolic response to injury and illness: 
estimation of energy and protein needs from indirect calorimetry and nitrogen balance. JPEN J Parenter Enteral Nutr 1979;3:452-6.

14. Ottenbacher KJ, Hsu Y, Granger CV, Fiedler RC. The reliability of the functional independence measure: a quantitative review. Arch Phys Med Rehabil 1996;77:1226-32.

15. Flodin L, Cederholm T, Saaf M, Samnegard E, Ekstrom W, Al-Ani AN, et al. Effects of protein-rich nutritional supplementation and bisphosphonates on body composition, handgrip strength and health-related quality of life after hip fracture: a 12-month randomized controlled study. BMC Geriatr 2015;15:149.

16. Yoshimura Y, Bise T, Shimazu S, Tanoue M, Tomioka Y, Araki M, et al. Effects of a leucine-enriched amino acid supplement on muscle mass, muscle strength, and physical function in post-stroke patients with sarcopenia: a randomized controlled trial. Nutrition 2019;58:1-6.

17. Yoshimura Y, Uchida K, Jeong S, Yamaga M. Effects of nutritional supplements on muscle mass and activities of daily living in elderly rehabilitation patients with decreased muscle mass: a randomized controlled trial. J Nutr Health Aging 2016;20:185-91.

18. Mishra S, Goldman JD, Sahyoun NR, Moshfegh AJ. Association between dietary protein intake and grip strength among adults aged 51 years and over: What We Eat in America, National Health and Nutrition Examination Survey 2011-2014. PLoS One 2018;13: e0191368.

19. Collins PF, Stratton RJ, Elia M. Nutritional support in chronic obstructive pulmonary disease: a systematic review and meta-analysis. Am J Clin Nutr 2012;95: 1385-95.

20. Takeuchi I, Yoshimura Y, Shimazu S, Jeong S, Yamaga M, Koga H. Effects of branched-chain amino acids and vitamin D supplementation on physical function, muscle mass and strength, and nutritional status in sarcopenic older adults undergoing hospital-based rehabilitation: a multicenter randomized controlled trial. Geriatr Gerontol Int 2019;19:12-7.

21. Wakabayashi H, Sakuma K. Rehabilitation nutrition for sarcopenia with disability: a combination of both rehabilitation and nutrition care management. J Cachexia Sarcopenia Muscle 2014;5:269-77.

22. Kokura Y, Wakabayashi H, Maeda K, Nishioka S, Nakahara S. Impact of a multidisciplinary rehabilitation nutrition team on evaluating sarcopenia, cachexia and practice of rehabilitation nutrition. J Med Invest 2017;64:140-5.

23. Nagano A, Nishioka S, Wakabayashi H. Rehabilitation nutrition for iatrogenic sarcopenia and sarcopenic dysphagia. J Nutr Health Aging 2019;23:256-65.

24. Tokunaga M, Mita S, Tashiro K, Yamaga M, Hashimoto Y, Nakanishi R, et al. Methods for comparing functional independence measure improvement degree for stroke patients between rehabilitation hospitals. Int J Phys Med Rehabil 2017;5:1000394. 